\title{
Pattern of Nailfold Capillaroscopy in Patients With Systemic Lupus Erythematosus
}

\author{
Ting ZHAO (i), Fu-an LIN(D, Hong-pu CHEN(D) \\ Department of Rheumatology and Immunology, Zhangzhou Affiliated Hospital of Fujian Medical University, Zhangzhou, China
}

\begin{abstract}
Objectives: This study aims to assess the nailfold capillary changes in patients with systemic lupus erythematosus (SLE), particularly among those with Raynaud's phenomenon (RP), and the correlation between nailfold capillary changes and autoantibodies and disease activity.

Patients and methods: A total of 85 patients (9 males, 76 females; median age 31 years; range, 15 to 58 years) with newly diagnosed SLE were selected between July 2016 and July 2018 from our hospital. Disease activity was scored by the SLE Disease Activity Index. Nailfold capillaroscopy (NFC) was performed in all patients.

Results: Normal pattern, non-specific pattern, and scleroderma pattern were found in $13(15.3 \%), 64$ (75.3\%), and eight (9.4\%) patients, respectively. There was no significant difference between anti-double stranded deoxyribonucleic acid, anti-Smith antibodies, and low complements (all $p>0.05$ ), while significant differences of NFC pattern were found between low disease activity and high disease activity $(p=0.002)$. RP was present in $31.7 \%$ of SLE patients, and the NFC findings in SLE patients with and without RP were significantly different in dilatation (81.5\% vs. $14.0 \%)$.

Conclusion: The results of our study showed that capillary changes were very common in patients with SLE, which seem to associate with disease activity and RP condition.

Keywords: Nailfold capillaroscopy, Raynaud's phenomenon, systemic lupus erythematosus.
\end{abstract}

Systemic lupus erythematosus (SLE) is considered a multi-factorial autoimmune disease, which produces a variety of autoantibodies and thus causes a series of complement cascade activation reaction. The clinical manifestations of SLE include Raynaud's phenomenon (RP), cutaneous involvement, arthritis, cardiac involvement, neurological involvement, vasculitis, hematologic involvement, periungual vasculitis, and renal involvement. The presence of autoantibodies and circulating immune complexes can cause vascular inflammation and damage the endothelial cells, leading to multiple organ dysfunctions. ${ }^{1}$ Nailfold, the skin wrinkle covering the root of the nail, allows investigation of patients' microcirculation. Recently, nailfold capillaroscopy (NFC) has attracted more attention because of its noninvasiveness, reproducibility, ease of operation, and low price. The microvascular changes can occur in early stages of some connective tissue diseases, which can be detected by NFC-related test, ensuring the possibility of early detection of disease. $^{2}$

Raynaud's phenomenon can be the earliest sign of systemic sclerosis (SSc) and other connective tissue diseases. Autoantibodies and capillaroscopic testing are generally accepted as valuable diagnostic tools to detect connective tissue diseases, particularly in patients with RP. ${ }^{3,4}$ Microvascular changes of SSc have been widely studied in recent years. In 2013, the American 
College of Rheumatology (ACR)/European League Against Rheumatism (EULAR) suggested new diagnosis criteria for $\mathrm{SSc}$, and for the first time, the NFC result was included in the diagnosis standard. ${ }^{5}$ NFC can be used to monitor and predict organ damage in the development of SSc, e.g. to predict the risk of pulmonary hypertension after the diagnosis of SSc. Furthermore, the abnormal level of nailfold capillary (particularly capillary deficiency) is correlated with disease activity. 6,7 Meanwhile, NFC can provide critical information in classifying primary and secondary RP. It has been reported that capillary structure of NFC testing for patients with primary RP was normal under non-cold stimulation, whereas secondary RP showed an abnormal capillary structure. ${ }^{8}$

Capillaroscopic changes can also be detected in SLE, Sjögren syndrome, undifferentiated connective tissue disease, inflammatory myopathy, and many other autoimmune diseases. However, studies in this field are still rather limited. Therefore, in this study, we aimed to assess the nailfold capillary changes in patients with SLE, particularly among those with RP, and the correlation between nailfold capillary changes and autoantibodies and disease activity.

\section{PATIENTS AND METHODS}

A total of 85 patients (9 males, 76 females; median age 31 years; range, 15 to 58 years) with newly diagnosed SLE who were admitted to Zhangzhou Affiliated Hospital of Fujian Medical University between May 2016 and May 2018 were recruited consecutively. The diagnostic criteria established by ACR/EULAR in 2009 were used to diagnose cases of SLE. The exclusion criteria included overlap syndrome, diabetes mellitus, pregnancy, or smoking behavior. Furthermore, patients who were unable to perform capillary evaluation, such as in the condition of very thick nailfold, more than four fingers injured or gangrene were excluded. The disease activity was scored by the SLE Disease Activity Index (SLEDAI) with a total scores of 105 points, which were divided into two groups: 0-11 points (inactive disease) and $\geq 12$ points (active disease). ${ }^{9,10}$ In all recruited patients, the SLEDAI scores ranged from 1-24 points. In order to divide study patients into two groups (low disease activity versus high disease activity) for comparisons with relevant large sample size, we chose the median value of SLEDAI score 12 as our cut-off point. The study protocol was approved by the Zhangzhou Affiliated Hospital of Fujian Medical University Ethics Committee. The study was conducted in accordance with the principles of the Declaration of Helsinki.

Patients were required to clean their fingers before the examination and stay under the room temperature $20-25^{\circ} \mathrm{C}$ for $15-20$ minutes. Ring finger and little finger of both hands were selected for capillaroscopy examination. In order to obtain high-resolution images, one-two drops of vegetable oil were smeared on the nailfold of screening finger before the test. The arm was required to be positioned at the same level as patient's heart. At least one image was taken for each finger.

The target area for recording was the distal layer of the capillaries under the papilla. The following indicators were recorded: capillary density (the numbers of capillaries counted in a $1 \times 1 \mathrm{~mm}$ area), avascular area (when $>2$ consecutive capillaries are missed), capillary length (normal or elongation: $\geq 300 \mu \mathrm{m}$ ), tortuous capillaries, capillary diameter including dilated capillaries $(20-50 \mu \mathrm{m})$ and megacapillaries (>50 $\mu \mathrm{m})$ branching (angiogenesis), erythrocyte aggregation and pericapillary hemorrhages..$^{11,12}$ Capillaroscopic pattern was defined as normal pattern (no changes were observed), non-specific (between the normal pattern and scleroderma pattern), and scleroderma pattern. ${ }^{13}$ According to the 2013 ACR/EULAR classification criteria, scleroderma pattern was defined as enlarged capillaries and/or capillary loss with or without pericapillary hemorrhages at the nailfold. Microcirculation detector, type TR8000D (TongRen Medical Co., Ltd., Jiangsu, China) with videocapillaroscope was used for the laboratorial analysis. All analyses were performed by the same investigator in a standard way.

\section{Statistical analysis}

Categorical variables were expressed in frequency and percentage, and the quantitative variables were described as mean and standard deviation. Comparisons between different subgroups of patients were conducted using chi-square tests for categorical data. A two-sided 
Table 1. Nailfold capillaroscopic pattern in patients with systemic lupus erythematosus

\begin{tabular}{|c|c|c|c|c|c|c|c|}
\hline \multirow[b]{2}{*}{ Study patients } & \multicolumn{2}{|c|}{ Normal } & \multicolumn{2}{|c|}{ Non-specific } & \multicolumn{2}{|c|}{ Scleroderma } & \multirow[b]{2}{*}{$p$} \\
\hline & $\mathrm{n}$ & $\%$ & $\mathrm{n}$ & $\%$ & $\mathrm{n}$ & $\%$ & \\
\hline All patients $(n=85)$ & 13 & 15.3 & 64 & 75.3 & 8 & 9.4 & - \\
\hline Anti-dsDNA ( $\mathrm{n}=75)$ & 11 & 14.7 & 58 & 77.3 & 6 & 8.0 & NS \\
\hline Anti-Sm ( $\mathrm{n}=54)$ & 9 & 16.7 & 40 & 74.1 & 5 & 9.26 & NS \\
\hline Hypocomplementemia $(\mathrm{n}=49)$ & 4 & 8.16 & 39 & 79.6 & 6 & 12.2 & NS \\
\hline Low disease activity $(n=57)$ & 11 & 19.3 & 45 & 78.9 & 1 & 1.75 & 0.002 \\
\hline High disease activity $(\mathrm{n}=28)$ & 2 & 7.14 & 19 & 67.9 & 7 & 25.0 & 0.002 \\
\hline
\end{tabular}

$p$ value less than 0.05 was considered to be statistically significant. Statistical analysis was performed by the IBM SPSS version 22.0 software (IBM corp., Armonk, New York, USA).

\section{RESULTS}

The duration of SLE disease ranged from three months to 10 years. Various SLE-related symptoms were detected in the recruited patients, including $\mathrm{RP}(\mathrm{n}=27,31.8 \%)$, cutaneous involvement $(\mathrm{n}=33,38.8 \%)$, arthritis $(\mathrm{n}=19,22.4 \%)$, cardiac involvement ( $n=6,7.1 \%)$, neurological involvement $(\mathrm{n}=8,9.4 \%)$, vasculitis $(\mathrm{n}=9,10.6 \%)$, hematologic involvement ( $\mathrm{n}=22,25.9 \%)$, and renal involvement $(\mathrm{n}=49,57.6 \%)$. Among these 85 patients, there were 75 patients $(88.2 \%)$ with positive result of anti-double stranded deoxyribonucleic acid (anti-dsDNA) antibodies, 54 patients (63.5\%) with positive anti-Smith (Sm) antibodies, and 49 patients $(57.6 \%)$ with hypocomplementemia (Table 1).

There were 57 patients (67.0\%) of inactive disease and 28 patients (32.9\%) of active disease. NFC was normal in 13 patients (15.3\%) while non-specific pattern and scleroderma pattern were observed in $64(75.3 \%)$ and eight patients (9.4\%), respectively. Of patients with positive anti-dsDNA antibodies, 14.7\%, 77.3\%, and 8.0\% showed normal pattern, non-specific pattern, and scleroderma pattern, respectively. Positive anti$\mathrm{Sm}$ antibodies were observed in $16.7 \%$ of patients with normal pattern, in $74.1 \%$ with non-specific pattern, and in $9.26 \%$ with scleroderma pattern. NFC of $8.16 \%$ of patients with low complement was normal, while $79.6 \%$ of patients had non-specific pattern and $12.2 \%$ presented a scleroderma-like pattern. No significant differences in laboratory parameters of the SLE patients were

Table 2. Nailfold capillaroscopy findings in systemic lupus erythematosus patients according to activity level of disease

\begin{tabular}{lcccccc}
\hline & \multicolumn{2}{l}{ High disease activity $(\mathrm{n}=28)$} & & \multicolumn{2}{l}{ Low disease activity $(\mathrm{n}=57)$} & \\
\cline { 2 - 3 } NFC findings & $\mathrm{n}$ & $\%$ & & $\mathrm{n}$ & $\%$ & \\
\hline Disturbed distribution & 10 & 35.7 & & 14 & 24.6 & 0.597 \\
Erythrocyte aggregation & 24 & 85.7 & & 40 & 70.2 & 0.096 \\
Anomalies & 14 & 50.0 & & 20 & 35.1 & 0.392 \\
Dilatation & 18 & 64.3 & & 14 & 24.6 & 0.541 \\
Megacapillaries & 5 & 17.9 & & 3 & 5.26 & 0.074 \\
Elongation & 18 & 64.3 & & 15 & 26.3 & 0.424 \\
Avascular area & 2 & 7.14 & & 3 & 5.26 & 0.535 \\
Hemorrhage & 23 & 82.1 & & 8 & 14.0 & $<0.005$ \\
\hline NFC: Nailfold capillaroscopy. & & & & & \\
\hline
\end{tabular}



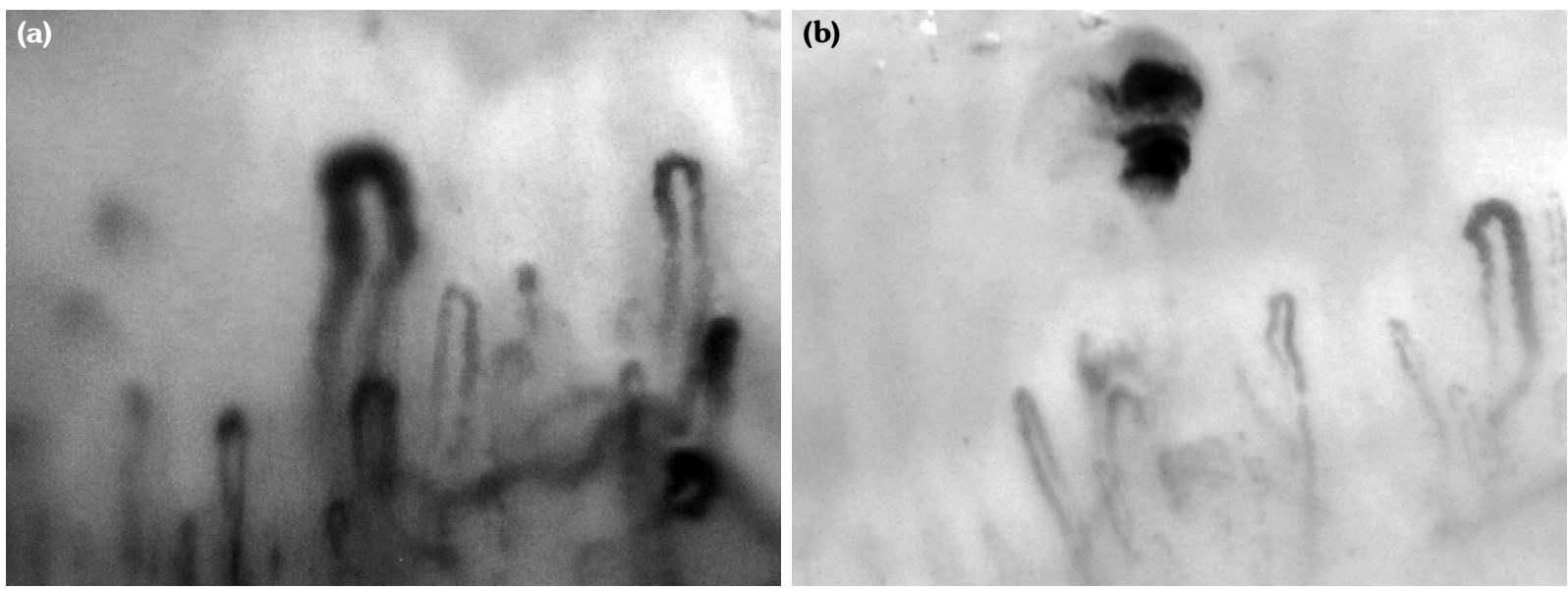

Figure 1. (a) Dilated capillaries $(20-50 \mu \mathrm{m})$, megacapillaries $(>50 \mu \mathrm{m})$ and erythrocyte aggregation in a patient with systemic lupus erythematosus. (b) Microhemorrhages and avascular area in a patient with systemic lupus erythematosus.

found between different capillaroscopic patterns ( $p>0.05$ ). The percentage of normal pattern was $19.3 \%$ in patients with inactive disease compared to $7.14 \%$ in patients with active disease. Also, rate of non-specific pattern was 78.9\% compared to $67.9 \%$, while rate of scleroderma pattern was $1.75 \%$ compared to $25.0 \%$ in patients with inactive disease compared to those with active disease, respectively. The incidence of almost all capillary abnormalities was higher in patients with active disease than that of patients with inactive disease and the difference was significant $(\mathrm{p}=0.002)$.

The NFC tests for SLE patients were also illustrated based on the groups of disease activity (Table 2). The prevalence of pericapillary hemorrhages was higher in patients with active disease $(82.1 \%)$ than those with inactive disease $(14.0 \%) \quad(p<0.005)$. However, no significant differences of distribution were observed for erythrocyte aggregation, anomalies, dilatation, giant loop, elongation, or avascular area between different disease activities (Figure 1).

The prevalence of dilated capillaries was $81.5 \%(\mathrm{n}=22)$ in the 27 SLE patients with $\mathrm{RP}$ as compared to $17.2 \% \quad(\mathrm{n}=10)$ in the 58 patients without RP $(p<0.005)$. No significant differences were seen in giant loop, avascular area or hemorrhage between the patients with and without RP (all p>0.05) (Table 3).

\section{DISCUSSION}

The current study demonstrated that the major NFC pattern in SLE patients was non-specific sub-types, followed by normal pattern and scleroderma pattern. Hemorrhage

Table 3. Nailfold capillaroscopic differences between systemic lupus erythematosus patients with and without Raynaud's phenomenon

\begin{tabular}{|c|c|c|c|c|c|}
\hline \multirow[b]{2}{*}{ NFC findings } & \multicolumn{2}{|c|}{$\mathrm{RP}(+)(\mathrm{n}=27)$} & \multicolumn{2}{|c|}{$\mathrm{RP}(-)(\mathrm{n}=58)$} & \multirow[b]{2}{*}{$p$} \\
\hline & $\mathrm{n}$ & $\%$ & $\mathrm{n}$ & $\%$ & \\
\hline Dilatation & 22 & 81.5 & 10 & 17.2 & $<0.005$ \\
\hline Megacapillaries & 5 & 18.5 & 3 & 5.17 & 0.063 \\
\hline Avascular area & 3 & 11.1 & 2 & 3.45 & 0.181 \\
\hline Hemorrhage & 15 & 55.6 & 16 & 27.6 & 0.572 \\
\hline
\end{tabular}


was associated with SLE's disease activity. No other characteristics of NFC were found to be associated with SLE disease activity. Meanwhile, distribution of dilatation was significantly higher in RP-positive patients.

Vascular abnormalities and inflammation are the major pathological changes of SLE. SLE patients are activated by autoimmune cells, leading to inflammation of vascular endothelial cells. Vascular endothelial cells produce endothelial adhesion factors through inflammation, which make the permeability of the vascular walls to increase, and then cause inflammatory cells to extravasate into the intertissue space. This series of processes has become the basis of vascular destruction and neovascularization. Abnormal changes can be seen in all of the blood vessels in SLE patients, including large, medium, and small ones. Many researchers have tried to find out the "full picture" of the systemic circulation and make a judgment of the disease by observing microcirculation through the nailfold. Lambova et al. ${ }^{14}$ reported that SLE patients showed abnormal NFC changes in length and diameter of the loop in most cases. It is worthy to note that although the current study only recruited newly diagnosed cases of SLE, some patients had been experiencing a few years of disease progression. This was mainly attributed to the fact that some patients had SLE-related symptoms a few years before visiting our hospital, while they did not undergo any clinical testing or treatment until diagnosis in our hospital.

The results of this study showed that SLE patients with positive anti-dsDNA/Sm antibodies and negative anti-dsDNA/Sm antibodies had a similar capillaroscopic pattern. Capillaroscopic findings among low complement and normal complement patients had been coincident, which indicated that there was no clear correlation between the changes of capillaroscopic and immunologic markers. Same conclusions had been reached by some scholars in the observation of capillaroscopic findings in inflammatory myopathy. ${ }^{15}$

In the current study, we found that abnormal capillary changes were very common being present in 72 out of 85 (84.7\%) SLE patients. Nailfold capillaroscopic pattern was significantly associated with SLE disease activity.
Capillaroscopic changes were related to SLEDAI scores and patients with inactive disease and active disease were remarkably different in scleroderma pattern. Patients with inactive SLE tended to have normal nailfold capillary, while active SLE patients tended to have abnormal pattern. These results are similar to many other studies that have reported a significant association between capillary abnormalities and disease activity in SLE. 9,16,17 More specifically, the prevalence of hemorrhage was higher in active SLE patients than inactive patients, which is consistent with other studies that indicated a greater frequency of disturbed distribution and hemorrhages in SLE patients with higher disease activity. ${ }^{18}$

Non-specific pattern was the most common pattern found in SLE patients while erythrocyte aggregation, dilatation, and anomalies were more common among them. Only an insignificant amount of SLE patients were observed to have scleroderma pattern and they were all accompanied by RP. Our results also showed that $\mathrm{RP}$ patients had higher frequency of dilatation compared to those without RP. Conclusions of this study are consistent with those studies from abroad. ${ }^{19,20}$ Abnormal NFC test results are commonly observed in SLE patients, but unlike SSc patients, most of the nailfold capillary changes in SLE are non-specific. ${ }^{20}$ The most important indicator of NFC is RP, while about $18-40 \%$ of SLE patients have accompanying RP. ${ }^{21}$ SLE patients with RP are more likely to have organ dysfunction than those without RP. ${ }^{22}$ A study investigated 79 SLE patients with RP and found that RP-positive patients were more likely to have central nervous system dysfunction and peripheral neuritis than RP-negative patients. ${ }^{21}$ In a follow-up study for children with SLE, authors reported higher risks of interstitial pneumonia and pulmonary hypertension for SLE patients with SSc pattern. ${ }^{16}$ Along with the treatment, the amount of abnormal nailfold capillaries decreased, suggesting that capillaroscopic changes could be an important indicator for the prognosis. RP may be the first warning sign occurring in SLE patients, and it has therefore been considered as a potential clinical predictor for SLE occurrence.

The limitations of this study are that capillaroscopy testing has high sensitivity and low specificity in detecting microvascular changes, and there is still a lack of standardized qualitative 
cut-off points for diagnosis. It is mainly used as a supplementary method in the clinics to describe microvascular changes. Another limitation is that the cross-sectional study design was limited in getting follow-up data for the recruited patients. Thus, we were unable to follow-up patients with positive antiphospholipid antibodies to confirm the diagnosis of antiphospholipid syndrome.

In conclusion, the current study revealed that abnormal NFC changes were common in SLE patients, which were correlated with SLE disease activity and RP condition. Further studies with larger sample sizes are needed to confirm this result.

\section{Declaration of conflicting interests}

The authors declared no conflicts of interest with respect to the authorship and/or publication of this article.

\section{Funding}

The current study is funded by Youth research project of Fujian Provincial Health Commission funding (No. 2018-2-39).

\section{REFERENCES}

1. Moneib HA, Salem SA, Aly DG, Khedr HT, Wafaey HA, Hassan HE. Assessment of serum vascular endothelial growth factor and nail fold capillaroscopy changes in systemic lupus erythematosus with and without cutaneous manifestations. J Dermatol 2012;39:52-7.

2. Etehad Tavakol M, Fatemi A, Karbalaie A, Emrani Z, Erlandsson BE. Nailfold Capillaroscopy in Rheumatic Diseases: Which Parameters Should Be Evaluated? Biomed Res Int 2015;2015:974530.

3. Ingegnoli F, Boracchi P, Gualtierotti R, Biganzoli EM, Zeni S, Lubatti $C$, et al. Improving outcome prediction of systemic sclerosis from isolated Raynaud's phenomenon: role of autoantibodies and nail-fold capillaroscopy. Rheumatology (Oxford) 2010;49:797-805.

4. Herrick AL, Moore TL, Murray AK, Whidby N, Manning JB, Bhushan M, et al. Nail-fold capillary abnormalities are associated with anti-centromere antibody and severity of digital ischaemia. Rheumatology (Oxford) 2010;49:1776-82.

5. van den Hoogen F, Khanna D, Fransen J, Johnson SR, Baron M, Tyndall A, et al. 2013 classification criteria for systemic sclerosis: an American College of Rheumatology/European League against Rheumatism collaborative initiative. Arthritis Rheum 2013;65:2737-47.
6. Voilliot D, Magne J, Dulgheru R, Kou S, Henri C, Caballero $\mathrm{L}$, et al. Prediction of new onset of resting pulmonary arterial hypertension in systemic sclerosis. Arch Cardiovasc Dis 2016;109:268-77.

7. Paxton D, Pauling JD. Does nailfold capillaroscopy help predict future outcomes in systemic sclerosis? A systematic literature review. Semin Arthritis Rheum 2018;48:482-94.

8. Lambova SN, Müller-Ladner $\mathrm{U}$. The role of capillaroscopy in differentiation of primary and secondary Raynaud's phenomenon in rheumatic diseases: a review of the literature and two case reports. Rheumatol Int 2009;29:1263-71.

9. Kuryliszyn-Moskal A, Ciolkiewicz M, Klimiuk PA, Sierakowski S. Clinical significance of nailfold capillaroscopy in systemic lupus erythematosus: correlation with endothelial cell activation markers and disease activity. Scand J Rheumatol 2009;38:38-45.

10. Fiechtner JJ, Montroy T. Treatment of moderately to severely active systemic lupus erythematosus with adrenocorticotropic hormone: a single-site, open-label trial. Lupus 2014;23:905-12.

11. Manfredi A, Sebastiani M, Cassone G, Pipitone N, Giuggioli D, Colaci M, et al. Nailfold capillaroscopic changes in dermatomyositis and polymyositis. Clin Rheumatol 2015;34:279-84.

12. Cutolo M, Sulli A, Smith V. How to perform and interpret capillaroscopy. Best Pract Res Clin Rheumatol 2013;27:237-48.

13. Cutolo M, Sulli A, Pizzorni C, Accardo S. Nailfold videocapillaroscopy assessment of microvascular damage in systemic sclerosis. $J$ Rheumatol 2000;27:155-60.

14. Lambova SN, Müller-Ladner U. Capillaroscopic pattern in systemic lupus erythematosus and undifferentiated connective tissue disease: what we still have to learn? Rheumatol Int 2013;33:689-95.

15. Selva-O'Callaghan A, Fonollosa-Pla V, TralleroAraguás E, Martínez-Gómez X, Simeon-Aznar $\mathrm{CP}$, Labrador-Horrillo $\mathrm{M}$, et al. Nailfold capillary microscopy in adults with inflammatory myopathy. Semin Arthritis Rheum 2010;39:398-404.

16. Ingegnoli F. Capillaroscopy abnormalities in relation to disease activity in juvenile systemic lupus erythematosus. Microvasc Res 2013;87:92-4.

17. Ciołkiewicz M, Kuryliszyn-Moskal A, Klimiuk PA. Analysis of correlations between selected endothelial cell activation markers, disease activity, and nailfold capillaroscopy microvascular changes in systemic lupus erythematosus patients. Clin Rheumatol 2010;29:175-80.

18. Kuryliszyn-Moskal A, Klimiuk PA, Sierakowski S, Ciołkiewicz M. Vascular endothelial growth factor in systemic lupus erythematosus: relationship to disease activity, systemic organ manifestation, and nailfold capillaroscopic abnormalities. Arch Immunol Ther Exp (Warsz) 2007;55:179-85. 
19. Higuera V, Amezcua-Guerra LM, Montoya H, Massó F, Patlán M, Paez A, et al. Association of Nail Dystrophy With Accrued Damage and Capillaroscopic Abnormalities in Systemic Lupus Erythematosus. J Clin Rheumatol 2016;22:13-8.

20. Shenavandeh S, Habibi S. Nailfold capillaroscopic changes in patients with systemic lupus erythematosus: correlations with disease activity, skin manifestation and nephritis. Lupus 2017;26:959-66.

21. Pavlov-Dolijanovic S, Damjanov NS, Vujasinovic Stupar NZ, Marcetic DR, Sefik-Bukilica MN, Petrovic RR. Is there a difference in systemic lupus erythematosus with and without Raynaud's phenomenon? Rheumatol Int 2013;33:859-65.

22. Hughes M, Herrick AL. Raynaud's phenomenon. Best Pract Res Clin Rheumatol 2016;30:112-32. 